\title{
ELABORACIÓN Y VALIDACIÓN DE UNA ESCALA DE MOTIVACIÓN Y ESTRATEGIAS DE APRENDIZAJE PARA ESCOLARES DE QUINTO Y SEXTO GRADO DE PRIMARIA
}

\author{
Jaime Aliaga Tovar \\ Magister en Psicología Educativa
}

\begin{abstract}
RESUMEN
Con base en el modelo de Pintrich y DeGroot (1990) y en estudios realizados en España por Roces et al. (1999), se ha elaborado una escala de motivación y estrategias de aprendizaje para utilizarse en el ámbito del tercer ciclo de educación primaria. La muestra estuvo compuesta por 1215 escolares repartidos proporcionalmente por tipo de colegio (estatal/privado), sexo y grado de estudios (quinto y sexto). El cuestionario tiene dos secciones, motivación y estrategias de aprendizaje, cada una con seis escalas.

La estructura interna fue evaluada por un análisis factorial exploratorio con componentes principales y rotación varimax. El análisis psicométrico hizo posible eliminar los itemes defectuosos y purificar las escalas, que muestran apropiados índices de validez y confiabilidad, constituyéndose un cuestionario útil para la evaluación de las referidas variables.
\end{abstract}

Palabras clave: Estrategias de aprendizaje, motivación, cuestionario, elaboración, educación primaria.

\section{ABSTRACT}

Based in the model of Printrich and DeGrott (1990) and in spanish studies by Roces et al (1999), it has been elaborated a Motivation and Strategy of learning Scale to be used in third cycle of elementary school. The sample was random and composed by 1215 students proportionally distributed according to kind of school (Public/private), sex and grade of studies $\left(5^{\text {th }}\right.$ or $\left.6^{\text {th }}\right)$ of elementary school. The questionnaire has 2 sections, motivation and strategy of learning, each one with 6 scales.

Its internal structure is evaluated by factor exploratory analysis with principal components and varimax rotation. This analysis along with item test correlations made possible to eliminate defective items and purifi the scales, which show psychometrically appropriate validity and reliability indexes, making of it an useful tool for the valuation of the referred variables.

Key Words: Learning Strategies, motivation, questionnaire, elaboration, elementary school.

Miembros: Carlos Ponce Díaz, Víctor Gutiérrez Olaya, Jorge Hidalgo Zerpa.

Colaboradores: Giovanna Pantoja Ruíz, Luis A Pinto López, Yesica Reyes Tejada. (Alumnos del sexto año de Psicología) 


\section{INTRODUCCIÓN}

Existen diversas teorías e interpretaciones sobre el aprendizaje humano. Mayer (1992) ha formulado algunas metáforas que expresan principios y consecuencias de varias de estas teorías e interpretaciones acerca del aprendizaje, especialmente del aprendizaje escolar. Una de estas metáforas: "el aprendizaje como construcción de significados" corresponde a una visión cognoscitiva del aprendizaje a la que subyace un paradigma emergente elaborado con los aportes teóricos de Piaget, Ausubel, Vigotsky, entre otros, que trata de las diversas formas de cómo aprendemos y enseñamos a apropiarnos integralmente de la realidad de un modo científico -aunque este no es el único modo, pues como bien señala Mendo (1999) los hay otros- tan válidos como este, por ejemplo, el filosófico, el estético, el religioso, y sobre todo el afectivo-. De acuerdo con esta metáfora, el aprendizaje es la construcción de significados; la enseñanza es la orientación del procesamiento cognitivo; el foco instruccional está centrado en el estudiante (procesamiento significativo); y los resultados, son cualitativos (estructura del conocimiento) (Mayer, 1992, en Beltrán, 1993: 16)

Esta metáfora expresa una concepción cognitiva constructiva que nos lleva a un nuevo modelo educativo y pedagógico. Este último, entendido como la representación simbólica, explicativa del proceso de enseñanza-aprendizaje que comprende lo curricular y lo didáctico, no está centrado ni en el profesor ni el en alumno, sino en el aprendizaje mismo.

En este modelo las actividades de ambos son diferentes. El profesor diseña actividades de aprendizaje, enseña a aprender, evalúa; el alumno, realiza actividades, construye su propio aprendizaje se autoevalúa. Pero a la vez el profesor no suministra conocimientos sino que participa en el proceso de construir conocimiento junto al estudiante; y el estudiante no se limita a adquirir conocimiento sino que lo construye usando la experiencia previa para comprender y moldear el nuevo aprendizaje. El papel del alumno en este sentido no es sólo activo sino proactivo y el conocimiento es construido y compartido por éste y el profesor, cuyo trabajo sería mediar en el encuentro del alumno con el conocimiento, orientando y guiando la actividad mental constructiva de sus alumnos, a quienes proporciona ayuda pedagógica ajustada a su competencia (Dávila, 2000; Díaz-Barriga \& Hernández, 1998), para producir un aprendizaje significativo y funcional. Significativo, cuando las ideas expresadas simbólicamente se relacionan de manera no arbitraria, sino sustancial (es decir, no al pie de la letra) con lo que el alumno ya sabe, y que se logra por el esfuerzo deliberado de éste por lograr tales relaciones y como producto de su implicación afectiva, de su actitud favorable, del querer aprender lo que se le presenta por considerarlo valioso (Ausubel, Novak \& Hasenian, 1983). Funcional, cuando puede utilizarse en una situación concreta para resolver un problema determinado, "utilización que puede extenderse al abordaje de nuevas situaciones para realizar nuevos aprendizajes" (Gómez, 1995, en Capella \& Sánchez Moreno, 1999: 95).

En consonancia con esta visión cognitiva constructiva del aprendizaje escolar en la que el alumno juega un papel no sólo activo sino también proactivo en su propio aprendizaje, pudiéndolo ajustar de acuerdo con sus propias necesidades y objetivos personales, él tiene que saber buscar, seleccionar, analizar, organizar, integrar en sus esquemas cognitivos y 
saber utilizar más tarde la información necesaria para afrontar con éxito las situaciones sociales en las que se vea inmerso, para lo cual requiere tener asimiladas un conjunto de estrategias de aprendizaje y su puesta en práctica.

Las estrategias de aprendizaje son definidas como secuencias integradas de procedimientos o actividades mentales activadas con el propósito de facilitar la adquisición, almacenamiento y/o utilización de la información (Nisbett \& Shucksmith, 1987) e implican un proceso de toma de decisiones consciente e intencional sobre que procedimiento de éstos utilizar para alcanzar determinados objetivos de aprendizaje (Monereo, 1993). En otras palabras, son un conjunto de pautas que desarrolla el estudiante para orientar de modo consciente e intencional sus actividades de aprendizaje, siendo producto de sus propias experiencias y de recomendaciones de compañeros o del profesor sobre cómo hacer más eficientes sus esfuerzos por aprender; constituyéndose en fundamentales para la calidad y éxito de las tareas académicas (Zimmerman \& MartínezPons, 1988; en Gonzáles-Cabanach y otros, 1999), puesto que inducen la actividad reflexiva del estudiante frente al material de estudio en las situaciones de enseñanza y por otra parte son elementos valiosos para el diseño, planificación, ejecución y evaluación de acciones pedagógicas, considerándolas algunos autores como aspectos centrales en el currículum educativo (Martínez \& Galán, 2000).

Se distinguen tres grandes grupos de estrategias: las estrategias cognitivas, las estrategias metacognitivas y las estrategias de administración de recursos, que se encuentran en continua interacción dinámica (Weinstein \& Mayer, 1986, en Valle, Gonzáles, Barca \& Núñez, 1996). Las primeras se refieren al conjunto de procesos que sirven de base a la realización de tareas intelectuales. Las segundas aluden al conocimiento del sujeto sobre el propio funcionamiento psicológico, sobre el aprendizaje; es decir, el ser consciente de lo que está haciendo, de tal manera que pueda controlar eficazmente sus propios procesos mentales. Las estrategias de administración de recursos, son caracterizadas como estrategias de apoyo.

Sin embargo, el conocimiento y puesta en práctica de estas estrategias es insuficiente para promover el aprendizaje escolar en el contexto del aula de clase, pues como sostienen Pintrich \& DeGroot (1990) - que al igual que otros psicólogos estadounidenses utilizan el constructo aprendizaje autoregulado (self regulated learning) que integra componentes cognitivos, metacognitivos, motivacionales y ambientales en la resolución de tareas académicas - los alumnos deben estar motivados para utilizar dichas estrategias así como regular su cognición y esfuerzo -. Es decir, los factores cognitivos y motivacionales y sus relaciones son los que ejercen una influencia más directa en la implicación del estudiante en el aprendizaje y en el rendimiento académico. En sus propias palabras: "Para tener buenos resultados académicos los estudiantes necesitan poseer tanto "habilidad" (skill) como "voluntad" (will) (Pintrich y DeGroot, 1990:38).

El modelo de Pintrich y DeGroot interrelaciona las estrategias y componentes motivacionales. Las estrategias son las siguientes: Estrategias cognitivas, que los estudiantes usan para aprender, recordar, entender, comprender el material; las estrategias metacognitivas del estudiante para planificar, monitorear y modificar su cognición; y las estrategias de administración del estudiante y el control de su esfuerzo sobre las tareas 
académicas. Los componentes motivacionales, por otro lado, son: un componente de expectancia, que responde a la pregunta ¿puedo hacer esta tarea? y que se conceptualiza en una variedad de maneras, por ejemplo: competencia percibida, autoeficacia, estilo atribucional y creencias de control, pero cuyo significado básico implica las creencias de los estudiantes de que se es capaz de resolver la tarea y de que se es responsable de su propio rendimiento. Un componente de Valor, que responde a la pregunta ¿por qué hago esta tarea? y que se refiere a las razones del alumno para hacer una tarea. Implica sus metas, sus creencias respecto a la importancia e interés de las tareas y actividades académicas. Incluye básicamente a las metas de aprendizaje vs. las metas de rendimiento y a la orientación intrínseca vs. la orientación extrínseca. Un componente afectivo, que responde a la pregunta ¿cómo me siento al hacer esta tarea? y que incluye un conjunto de reacciones emocionales del alumno ante la tarea que pueden ser relevantes, por ejemplo, orgullo, ira, ansiedad, culpa, pero "en el aprendizaje escolar una de las más importantes es la ansiedad ante la prueba" (Wigfield \& Eccles, 1988, en Pintrich \& De Groot, 1990: 34).

La valoración de estos aspectos se ha tomado imprescindible en la visión cognitiva mediacional. Para nuestra investigación esta valoración requiere previamente decidir en que nivel evolutivo del alumno sería conveniente realizarla y el cómo hacerla. En cuanto a lo primero, la adquisición de las estrategias básicas de aprendizaje tiene una edad crítica para su enseñanza que va de los 11 a los 14 años de edad, que corresponden en parte al quinto y sexto grados de la educación primaria; período que coincide con el desarrollo de estructuras cognitivas previas a la consolidación del pensamiento formal propio del adolescente, y en el cual resultan más favorables: 1) el entrenamiento en estrategias de aprendizaje "ya que es cuando aparece el pensamiento formal, siendo esta la razón por la que se aplican la mayor parte de los métodos de enseñar a pensar a esta edad" (Beltrán, 1983: 53) y, 2) la orientación de los aspectos motivacionales del estudiante implicados en las situaciones de aprendizaje, con miras a su asimilación como componentes duraderos de la actividad intelectual del estudiante a lo largo de los siguientes niveles educativos.

En cuanto a lo segundo, la evaluación puede realizarse mediante informes verbales, observación o mediante escalas o cuestionarios. Estos últimos, asumiendo que el respondiente es capaz de describir correctamente su propia conducta, que sus reactivos son muestras de conducta aceptados después de un análisis empírico-estadístico que establece su contribución en cierto grado a la medida de la dimensión o característica evaluada, son los instrumentos más utilizados con este fin en situaciones educativas en que es necesario realizar una prospección amplia de un colectivo numeroso.

Se han elaborado diversas escalas que evalúan la motivación y estrategias de aprendizaje, por ejemplo, el LASSI (Weinstein \& Mayer, 1990) en EEUU, y el ACRA (Román y Gallego, 1994) en España. De hecho Pintrich y DeGroot (1990) han elaborado el MSLQ (Motivated Strategies for Learning Questionnaire) basado en el modelo que proponen y que es aplicable a alumnos desde el primer año de educación secundaria, cuya versión española con algunas modificaciones al modelo en base a evidencia empírica, es el CEAM (Roces, Gonzáles Pienda, Núñez, Gonzáles-Pumariega, García \& Alvarez, 1999). En nuestro país, teniendo en cuenta la adaptación y estandarización del ACRA en el nivel secundario y en colegios particulares (Cano, 1997), no existen instrumentos que evalúen 
estos aspectos y menos en el nivel primario.

En este contexto, nos proponemos abordar la elaboración de un cuestionario de estrategias motivadas de aprendizaje para los grados quinto y sexto de primaria.

\section{Objetivo}

Construir un cuestionario válido y confiable para evaluar la motivación y estrategias de aprendizaje basado en el modelo de Pintrich y DeGroot (1990) y en los estudios españoles de este modelo (Roces, et. at., 1999, 1997), en la población escolar del quinto y sexto grado de primaria de Lima Metropolitana.

\section{Método}

Tipo de investigación. Es el aplicativo porque se orienta al uso de manera práctica de conocimiento teórico sobre las variables implicadas: motivación, estrategias de aprendizaje y mensuración psicológica; en este último sentido es fundamentalmente psicométrica porque está orientada a crear un instrumento de medición psicológica (Alarcón, 1991: 234).

Diseño. Es transeccional porque se recolectan los datos en un solo momento, en un tiempo único, y también es correlacional porque busca establecer relaciones entre variables medidas, en un momento determinado en un grupo de personas (Hemández, Femández \& Baptista, 1998), es decir, correlaciones entre los diferentes reactivos y variables tenidos en cuenta para el análisis de los ítemes y el análisis factorial, cuyos resultados serán utilizados para analizar las características psicométricas del instrumento elaborado.

Población. Es la de escolares varones y mujeres que cursan el quinto y sexto grados de primaria de menores en colegios estatales y privados de las Unidades de Servicios Educativos (USES) de Lima Metropolitana. En el quinto grado esta población es de 140 285 (107 898 de centros estatales; 32296 de colegios privados); en el sexto grado es de 126758 (100427 de colegios estatales; 26331 de colegios privados). En la población, los escolares varones y mujeres se hallan en número similar.

Muestra. Teniendo como marco de muestreo a las Unidades de Servicios Educativos (USES) de Lima, la muestra se obtuvo por un muestreo probabilístico polietápico por conglomerados, seleccionando a las unidades primarias de muestreo (colegios) y a las unidades secundarias de muestreo (secciones) de forma aleatoria simple y de estás a las unidades últimas (individuos) por cuota de sexo. El tamaño mínimo de la muestra fue de 625 , número calculado para estimar los parámetros poblacionales con un nivel de confianza del $95 \%$ y un error muestral de $+/-4 \%$, utilizando las tablas de Arkin y Colton (1962, citados en Sierra Bravo, 1994). La muestra final fue de 1215 sujetos, obtenida de 18 colegios estatales y privados y estratificada por afijación proporcional de modo que represente a la población por sexo, grado y tipo de centro educativo.

Procedimiento. Se elaboró el cuestionario de estrategias motivadas de aprendizaje para el quinto y sexto grados de primaria (EAM-56P) siguiendo procedimientos y normas que en 
sus aspectos básicos son usuales en la elaboración de instrumentos de medida psicométricos:

1) Se elaboró el marco teórico de la prueba con base en el modelo de Pintrich y De Groot (1990) y en los estudios españoles de este modelo, que se sustenta en una visión cognitiva general de la motivación y las estrategias de aprendizaje, y se operacionaliza en el MSLQ (Motivated Strategies for Leaming Questionnaire), cuyos antecedentes primigenios datan de 1982 y que se desarrolló formalmente desde 1986 en la Universidad de Michigan con estudiantes de pregrado que seguían el curso "Aprendiendo a aprender" . El MSLQ tiene dos secciones: motivación (seis escalas en 31 ítemes) y sección estrategias de aprendizaje (31 ítemes), que incluye además 19 ítemes concernientes a la administración por el estudiante de diferentes recursos. Las escalas son nueve. En total son 81 reactivos que se responden en una escala del 1 al 7 (Pintrich, Smith, García \& McKeachie, 1991). La adaptación española del MSLQ, el CEAM, tiene seis escalas motivacionales y siete de estrategias de aprendizaje.

2) Sustentados en este marco se definió conceptualmente cada una de las estrategias y dimensiones motivacionales, las que conforman escalas independientes.

3) En cuanto al formato, los ítemes fueran redactados teniendo en cuenta la adecuación semántica y sintáctica de sus contenidos a las características de la población. La estructura de las alternativas de respuesta fue diseñada con tres opciones: A. "Si, me pasa eso, es verdad" B. "A medias, a veces sí a veces no; C. No, no me pasa eso, es mentira". Por último, el cuestionario fue impreso en cuatro colores (azul, rojo, naranja y verde) con el fin de hacerlo más atrayente.

4) Una primera versión del cuestionario compuesta de 100 reactivos fue administrada a 45 alumnos del curso de Metodología de la Investigación 11 del turno nocturno (ciclo VIII, semestre 200 1-2) de la Facultad de Psicología de la UNMSM, quienes realizaron un conjunto de apreciaciones acerca de la pertinencia y redacción de los reactivos. Con base en sus sugerencias varios reactivos fueron eliminados y otros reformulados, quedando el cuestionario compuesto por 85 reactivos en esta fase.

5) La versión resultante fue sometida a un proceso de validez de contenido atendiendo al criterio de cinco jueces, quienes son reconocidos psicólogos educativos y ampliamente conocedores del tema. La concordancia de los jueces fue evaluada por el coeficiente $\mathrm{V}$ de Aiken con un nivel de significación del 0.05. En esta fase no se eliminó a ninguno de los elementos del cuestionario.

La descripción del contenido de las escalas es la siguiente:

\subsection{Sección motivación.}

a) Componente de expectancia: Autoconfianza para el aprendizaje y el rendimiento (8 ítems). Los reactivos evalúan dos aspectos de las expectativas: expectativas por el 
éxito y autoeficacia. La primera refiere a las expectativas por el rendimiento, y se relaciona específicamente con el rendimiento en tareas. La autoeficacia incluye juicios acerca de la propia habilidad para realizar bien las tareas y la confianza en las habilidades personales para rendir en las tareas.

b) Componente de expectancia: Creencias sobre el control del aprendizaje (5 ítems). Refiere a las creencias de los estudiantes acerca de que sus esfuerzos para aprender tendrán un resultado positivo. Creencias de que este rendimiento es contingente con el propio esfuerzo en contraste con otros factores externos, como podría ser el profesor. Refleja hasta que punto el estudiante cree que su dominio de su rendimiento depende de su propio esfuerzo y su modo de estudiar.

c) Componente de valor: Orientación a metas internas (5 ítems). Valora el grado en que el estudiante considera que toma parte en una tarea por razones como el reto, la curiosidad y la maestría o dominio. Con acuerdo a esta orientación motivacional, la participación del estudiante es un fin en sí mismo. Está orientado a la tarea.

d) Componente de valor: Orientación a metas externas (5 ítems). Se refiere al grado en que el estudiante percibe que participa de una tarea por razones como las notas, premios, la opinión de otros o el superar a sus compañeros. Esta orientado al resultado. Cuando una persona tiene una alta orientación en este sentido su implicación en las tareas es un medio para conseguir objetivos extrínsecos a la propia naturaleza de la tarea.

e) Componente de valor: Valor de la tarea (6 ítems). Refiere a la evaluación del estudiante, lo que piensa, acerca del interés, importancia y utilidad que tiene la tarea.

f) Componente afectivo: Ansiedad ante la prueba. Esta negativamente relacionada con las expectativas y con el rendimiento académico. Tiene dos componentes: cognitivo y emocional. El cognitivo concierne a la preocupación que es una fuente de de cremento del rendimiento. Escala conformada por cuatro ítems de la adaptación peruana del IDASE (Inventario de ansiedad ante los exámenes) (Aliaga, Ponce, Bernaola y Pecho, 2000).

\subsection{Sección Estrategias de aprendizaje.}

a) Componente estrategias cognitivas y metacognitivas: Elaboración (9 ítems). Esta escala se refiere a sí el alumno aplica conocimientos anteriores a situaciones nuevas para resolver problemas, tomar decisiones o hacer evaluaciones críticas, y si establece conexiones de la información nueva con la que ya tenía para recordarla mejor; incluye, por ejemplo, el realizar resúmenes, elaborar notas.

b) Componente estrategias cognitivas y metacognitivas: Organización ( 9 ítems). Estas estrategias ayudan al alumno a seleccionar apropiadamente la información y a construir conexiones entre la información para ser aprendida. Hace referencia a las estrategias que emplea el alumno para acometer el estudio de la materia y seleccionar la información relevante, tales como, por ejemplo, esquemas, resúmenes, subrayado.

c) Componente estrategias cognitivas y metacognitivas: Autorregulación metacognitiva (14 ítems). Se refiere a la autoconciencia y el control de la cognición; al planeamiento, monitoreo y regulación; en otras palabras, al 
establecimiento de metas y a la regulación del estudio y de la propia comprensión.

d) Componente estrategias de administración de recursos: Administración del tiempo (9 ítems). Envuelve el planeamiento y administración del tiempo propio de estudio, el uso efectivo de este en el logro de objetivos realistas.

e) Componente estrategias de administración de recursos: Constancia (7 ítemes). Esta escala refleja la diligencia y esfuerzo para llevar al día las actividades y trabajos de las diferentes asignaturas y alcanzar las metas establecidas.

f) Componente estrategias de administración de recursos: Búsqueda de ayuda (4 ítems). Un aspecto del medio ambiente que el estudiante debe aprender a manejar es el soporte y apoyo de otros: alumnos, el profesor y otras personas significativas.

6) Se administró el cuestionario colectivamente al alumnado, dentro de sus grupos de clase, por psicólogos y colaboradores cuidándose rigurosamente las condiciones de la aplicación con la finalidad que las respuestas se relacionen con las variables en estudio.

7) Teniendo en cuenta que la validez es el resultado de un proceso largo, complejo y en permanente revisión que se define por a) la extensión con la que un test mide un rasgo subyacente específico hipotético o constructo y, b) en conexión con esta amplitud por la relación de sus puntajes con una medida de criterio externo; con un enfoque intrapruebas se estimo la validez de constructo dando respuesta a la pregunta ¿qué proporción de la varianza o variabilidad de las puntuaciones de los sujetos de la muestra en la escala se puede atribuir a la variable que mide? Esta estimación se realizó en cada una de las escalas construidas con base en la información del análisis de su estructura por medio de un análisis factorial exploratorio con la técnica de los componentes principales y rotación varimax, comprobándose previamente la idoneidad de las matrices de correlaciones de los ítems de las escalas para ser sometidas al análisis factorial mediante el test de esfericidad de Bartlett y el índice de adecuación de muestreo (KMO). En cuanto a las cargas o saturaciones (loading) de los reactivos se estableció que debían ser cuando menos de 0.30 con el factor.

8) La confiabilidad de las escalas se estableció averiguando en cada una de ellas su consistencia interna grado de intercorrelación y de equivalencia de sus ítems, utilizando el coeficiente alfa de Crombach. Se establecieron los siguientes criterios de aceptabilidad: a) las escalas debían tener como mínimo tres ítems; b) los ítems debían contribuir a la consistencia interna de la escala (correlación item-total corregida no inferior a 0.20), evaluada por el coeficiente alfa de Crombach.

\section{Resultados y discusión}

Como ya se anotó, la validez de contenido para cada una de las escalas fue evaluada por cinco jueces, siendo valorado su grado de concordancia con la prueba V de Aiken, cuyos resultados tuvieron un valor mínimo de 0.80 y un valor máximo de 0.90 , significativos al nivel del 0.05 . En este contexto, puede asumirse que los reactivos constituyen una muestra 
representativa del dominio o universo temático tal como este fue definido para cada una de las escalas y, en este sentido, puede concluirse que éstas poseen validez de contenido.

Los resultados que se detallan y analizan a continuación corresponden a las escalas con los reactivos que quedaron después del cruce de información resultante de los diversos análisis estadísticos y psicométricos realizados.

\subsection{Sección Motivación.}

\subsubsection{Componente de expectancia.}

Tabla 1

Componente de Expectancia: Autoconfianza para el aprendizaje y el rendimiento.

\begin{tabular}{|c|c|c|c|c|c|c|c|}
\hline $\begin{array}{l}\text { Índice } \\
\text { KMO }\end{array}$ & $\begin{array}{l}\text { Test de } \\
\text { Bartlett } \\
\text { ( sig) }\end{array}$ & Factores & $\begin{array}{c}\% \\
\text { varianza } \\
\text { explicada }\end{array}$ & Items & Saturación & rit & Alfa \\
\hline 0.781 & 0.001 & 1 & 38.187 & $\begin{array}{c}\text { A1 } \\
\text { A8 } \\
\text { A15 } \\
\text { A19 } \\
\text { A24 } \\
\text { A27 }\end{array}$ & $\begin{array}{l}0.419 \\
0.618 \\
0.648 \\
0.638 \\
0.615 \\
0.632\end{array}$ & $\begin{array}{l}0.378 \\
0.405 \\
0.446 \\
0.407 \\
0.388 \\
0.402\end{array}$ & 0.675 \\
\hline \multicolumn{8}{|c|}{ Componente de expectancia : Control de Creencias en el Aprendizaje } \\
\hline $\begin{array}{l}\text { Índice } \\
\text { KMO }\end{array}$ & $\begin{array}{l}\text { Test de } \\
\text { Bartlett } \\
\text { (sig) }\end{array}$ & Factores & $\begin{array}{c}\% \\
\text { varianza } \\
\text { explicada }\end{array}$ & Items & Saturación & rit & Alfa \\
\hline 0.607 & 0.001 & 1 & 51.979 & $\begin{array}{l}\text { A13 } \\
\text { A20 } \\
\text { A31 }\end{array}$ & $\begin{array}{l}0.716 \\
0.768 \\
0.676\end{array}$ & $\begin{array}{l}0.342 \\
0.394 \\
0.311\end{array}$ & 0.536 \\
\hline
\end{tabular}

Los datos de la tabla 1 indican que en ambas escalas hay una sola variable latente o factor que subyace a sus reactivos. Ambos factores son estadísticamente significativos como lo indica la media de las saturaciones de los ítems que les dan estructura: 0.595 en Autoconfianza para el aprendizaje y el rendimiento; y 0.72 para Creencias de control en el aprendizaje; además, ambas escalas tienen la totalidad o la gran mayoría de sus ítems con cargas no inferiores a 0.50 que propician una clara definición e interpretación factorial (Comrey, 1985). En un nivel individual, ambas escalas tienen índices de discriminación (rit) indicadores de correlaciones estadísticas muy significativas entre cada ítem y el total $(\mathrm{p}<0.001)$. Apreciando la totalidad de las escalas, los coeficientes alfa son relativamente bajos, pero aceptables por el número de sus reactivos (Morales, 1988), sobre todo en la escala Creencias de control en el aprendizaje. 
1.1.2 Componente de valor.

Tabla 2

Componente de Valor: Orientación a metas internas

\begin{tabular}{|c|c|c|c|c|c|c|c|}
\hline $\begin{array}{l}\text { Indice } \\
\text { KMO }\end{array}$ & $\begin{array}{c}\text { Test de } \\
\text { Bartlett } \\
\text { ( sig) }\end{array}$ & Factores & $\begin{array}{c}\% \\
\text { varianza } \\
\text { explicada }\end{array}$ & Items & Saturación & rit & Alfa \\
\hline 0.654 & 0.001 & 1 & 40.479 & $\begin{array}{l}\text { A2 } \\
\text { A9 } \\
\text { A11 } \\
\text { A35 }\end{array}$ & $\begin{array}{l}0.600 \\
0.597 \\
0.655 \\
0.688 \\
\end{array}$ & $\begin{array}{l}0.277 \\
0.273 \\
0.313 \\
0.338 \\
\end{array}$ & 0.508 \\
\hline \multicolumn{8}{|c|}{ Componente de Valor: Orientación a metas externas } \\
\hline $\begin{array}{l}\text { Índice } \\
\text { KMO }\end{array}$ & $\begin{array}{c}\text { Test de } \\
\text { Bartlett } \\
\text { (sig) }\end{array}$ & Factores & $\begin{array}{c}\% \\
\text { varianza } \\
\text { explicada }\end{array}$ & Items & Saturación & rit & Alfa \\
\hline 0.550 & 0.001 & 2 & 61.229 & $\begin{array}{c}\text { Al6 (Fl) } \\
\text { A22 (Fl) } \\
\text { A4 (F2) } \\
\text { A30 (F2) }\end{array}$ & $\begin{array}{c}0.731 \\
0.796 \\
0.782 \\
0.763\end{array}$ & $\begin{array}{l}0.251 \\
0.238 \\
0.199 \\
0.201\end{array}$ & 0.396 \\
\hline \multicolumn{8}{|c|}{ Componente de Valor: Valor de la tarea } \\
\hline $\begin{array}{l}\text { Índice } \\
\text { KMO }\end{array}$ & $\begin{array}{c}\text { Test de } \\
\text { Bartlett } \\
\quad \text { (sig) }\end{array}$ & Factores & $\begin{array}{c}\% \\
\text { varianza } \\
\text { explicada }\end{array}$ & Items & Saturación & rit & Alfa \\
\hline 0.687 & 0.001 & 1 & 43.315 & $\begin{array}{l}\text { A14 } \\
\text { A17 } \\
\text { A23 } \\
\text { A29 }\end{array}$ & $\begin{array}{l}0.626 \\
0.556 \\
0.690 \\
0.620\end{array}$ & $\begin{array}{l}0.378 \\
0.282 \\
0.438 \\
0.390\end{array}$ & 0.593 \\
\hline
\end{tabular}

Los datos sumarizados en la tabla 2 indican que en los reactivos de las escalas Orientación a metas internas y Valor de la tarea subyace un solo factor que explican cantidades apreciables de varianza. Estos factores por la saturación media de sus elementos son estadísticamente significativos: 0.635 para Orientación a metas internas y 0.623 para Valor de la tarea. Por otro lado, ambas escalas tienen una clara definición e interpretación factorial pues sus saturaciones son en ambos casos superiores a 0.50 (Comrey, 1985). En un plano singular, las dos escalas tienen un promedio de sus índices de discriminación o correlaciones ítem test corregidas (rit) estadísticamente muy significativos (0.303 para Orientación a metas internas y 0.372) para valor de la tarea. Sin embargo, estos promedios son inferiores a las escalas de la Tabla 1 . Los coeficientes alfa, por su lado, alcanzan valores relativamente bajos, pero como en el caso anterior aceptables por el número de sus reactivos (Morales, 1988). En lo concerniente a la escala Orientación a metas externas las cifras de los índices calculados indican que es la escala más débil del conjunto (y de todas 
ellas).

El análisis factorial indica que es una escala factorialmente compleja y que la variabilidad de las respuestas de los sujetos a sus ítems se explican por dos factores que si bien en conjunto explican un porcentaje apreciable de varianza (la más alta de todas las escalas: 61.229\%) con saturaciones que propician una clara defmición factorial, la calidad individual de sus reactivos expresada en sus índices de discriminación es relativamente pobre, con dos de sus elementos bordeando el rit mínimo de 0.20, configurándose en consecuencia un coeficiente alfa (0.396), es decir una consistencia interna bastante baja.

\subsubsection{Componente afectivo.}

Tabla 3

Componente afectivo: Ansiedad ante la prueba

\begin{tabular}{|lccccccc|}
\hline $\begin{array}{l}\text { Indice } \\
\text { KMO }\end{array}$ & $\begin{array}{c}\text { Test de } \\
\text { Bartlett } \\
\text { (sig) }\end{array}$ & Factores & $\begin{array}{c}\text { \% } \\
\text { varianza } \\
\text { explicada }\end{array}$ & ltems & Saturación & rit & Alfa \\
\hline 0.683 & 0.000 & 1 & 43.335 & A7 & 0.656 & 0.345 & \\
& & & & A10 & 0.677 & 0.361 & 00.563 \\
& & & & A26 & 0.613 & 0.311 & \\
& & & & A22 & 0.685 & 0.370 & \\
\hline
\end{tabular}

Los cuatro ítems de esta escala pertenecen al IDASE (Inventario de ansiedad ante los exámenes) de Bauermeister, Collazo y Spielberger (1983), dos a la escala de emocionalidad y dos a la escala de preocupación; fueron incluidos por sus adecuados índices psicométricos obtenidos en el estudio de adaptación realizado en Perú (Aliaga, Ponce, Bernaola\& Pecho, 2001). El análisis factorial indica la existencia de un solo factor que explica el $43.335 \%$ de la varianza de las puntuaciones de los sujetos en la escala, que puede ser calificado de bueno en comparación al 40.292\% que explicaban tres factores en el IDASE. El factor esta factorialmente bien definido porque los reactivos tienen cargas superiores a 0.60 , por otro lado, sus índices de discriminación (promedio: 0.346) son inferiores a los hallados en la citada adaptación (mediana: 0.513), probablemente por las características de la muestra que pueden sintetizarse en una inferioridad cronológica de aproximadamente cinco años de edad entre la muestra en estudio y la muestra de adaptación del referido inventario (quinto año de secundaria).

Tabla 4 1.1.4 Sección motivación: Intercorrelación de las escalas

\begin{tabular}{|lcccccc|}
\hline & Autoconfi & Creen Con & Orieninterna & Orienxterna & Valor tarea & Ansiedad \\
\hline Autoconfi & ----------- & 0.033 & $0,366^{* *}$ & $0,290^{* *}$ & $0,360^{* *}$ & -0.089 \\
Creen Con & --------- & $0,060^{*}$ & $0,083^{* * *}$ & $0,103^{* *}$ & $0,2022^{* *}$ \\
Orieninterna & & ----------- & 0.298 & $0,333^{* *}$ & 0.066 \\
Orieexterna & & & --------- & $0,244^{* *}$ & $0,310^{* *}$ \\
Valor tarea & & & & ---------- & 0.011 \\
Ansiedad & & & & & -------- \\
\hline
\end{tabular}

$*(\mathrm{p}<0,05) * *(\mathrm{p}<0,01) ; \mathrm{N}=1215$ 


\subsection{Sección estrategias de aprendizaje.}

\subsubsection{Estrategias cognitivas y metacognitivas}

Tabla 5

Componente estrategias cognitivas y metacognitivas: Elaboración

\begin{tabular}{|c|c|c|c|c|c|c|c|}
\hline $\begin{array}{l}\text { Índice } \\
\text { KMO }\end{array}$ & $\begin{array}{c}\text { Test de } \\
\text { Bartlett (sig) }\end{array}$ & Factores & $\begin{array}{l}\% \text { varianza } \\
\text { explicada }\end{array}$ & Items & Saturación & rit & alfa \\
\hline \multirow[t]{6}{*}{0.767} & \multirow[t]{6}{*}{0.001} & \multirow[t]{6}{*}{1} & \multirow[t]{6}{*}{36.419} & A34 & 0.587 & 0.369 & \multirow{6}{*}{0.65} \\
\hline & & & & A40 & 0.577 & 0.359 & \\
\hline & & & & A49 & 0.663 & 0.431 & \\
\hline & & & & A53 & 0.603 & 0.376 & \\
\hline & & & & A 82 & 0.609 & 0.381 & \\
\hline & & & & A84 & 0.578 & 0.357 & \\
\hline \multicolumn{8}{|c|}{ Componente estrategias cognitivas y metacognitivas :Organización } \\
\hline $\begin{array}{l}\text { Índice } \\
\text { KMO }\end{array}$ & $\begin{array}{c}\text { Test de } \\
\text { Bartlett (sig) }\end{array}$ & Factores & $\begin{array}{l}\text { \% varianza } \\
\text { explicada }\end{array}$ & Items & Saturación & rit & alfa \\
\hline \multirow[t]{6}{*}{0.784} & 0.001 & 1 & 39.154 & A55 & 0.675 & 0.464 & \multirow{6}{*}{0.687} \\
\hline & & & & A61 & 0.601 & 0.394 & \\
\hline & & & & A66 & 0.674 & 0.465 & \\
\hline & & & & A58 & 0.577 & 0.371 & \\
\hline & & & & A47 & 0.603 & 0.399 & \\
\hline & & & & A80 & 0.618 & 0.411 & \\
\hline \multicolumn{8}{|c|}{ Componente estrategias cognitivas y metacognitivas : Autorregulacion metacognitiva } \\
\hline $\begin{array}{l}\text { Índice } \\
\text { KMO }\end{array}$ & $\begin{array}{c}\text { Test de } \\
\text { Bartlett (sig) }\end{array}$ & Factores & $\begin{array}{l}\% \text { varianza } \\
\text { explicada }\end{array}$ & Items & Saturación & rit & alfa \\
\hline \multirow[t]{8}{*}{0.807} & 0.001 & 2 & 43.798 & A39(F1) & 0.632 & 0.372 & \multirow{8}{*}{0.675} \\
\hline & & & & A46(F1) & 0.648 & 0.454 & \\
\hline & & & & $\mathrm{A} 47(\mathrm{~F} 1)$ & 0.575 & 0.428 & \\
\hline & & & & A59(F1) & 0.681 & 0.451 & \\
\hline & & & & A76(F1) & 0.566 & 0.281 & \\
\hline & & & & A38(F2) & 0.61 & 0.289 & \\
\hline & & & & A64(F2) & 0.71 & 0.329 & \\
\hline & & & & $\mathrm{A} 81(\mathrm{~F} 2)$ & 0.697 & 0.341 & \\
\hline
\end{tabular}

La intercorrelación de las escalas es otro modo de observar la validez. Los coeficientes de correlación pueden analizarse con base en dos orientaciones: significatividad estadística y tamaño o cuantía. En lo concerniente a la significatividad, la mayoría de los coeficientes son significativos al nivel del 0.01 , indicando con ello que comparten cierta varianza; pero en cuanto a su tamaño, el más elevado ( $\mathrm{r}=0.366$ entre Autoconfianza y Orientación interna) apenas puede ser calificado de "correlación débil" (Elorza, 1987). En todo caso, estos coeficientes señalan que las escalas evalúan aspectos independientes y psicológicamente significativos.

Los resultados mostrados en la tabla 5 indican en el caso de las escalas Elaboración y Organización que a sus reactivos les subyace un único factor, que explica cantidades relativamente apreciables de la variabilidad de sus puntuaciones. Factores que como casi la totalidad de los que hemos analizado hasta aquí son estadísticamente significativos dado el promedio de sus, saturaciones o loadings: 0.602 para Elaboración, 0.624 en el caso de 
Organización, lo que factorialmente los hace claros para su interpretación (saturaciones mayores a 0.50). Individualmente las dos escalas tienen índices de discriminación (rit) con promedios estadísticamente muy significativos ( $\mathrm{r}=0.378$ Elaboración; 0.417 Organización). Los coeficientes alfa son de los más elevados hasta aquí, aunque relativamente bajos pero de cuantía aceptable en vista del número de sus elementos. Por último, la escala Autoregulación de la metacognición por su propia naturaleza poco desarrollada en las edades de la muestra de estudio, se presenta como factorialmente compleja. A la variabilidad de sus puntuaciones le subyacen dos factores, pero a diferencia de la escala de Orientación de metas externas o extrínsecas, sus saturaciones (promedio: 0.639) indican una definición factorial clara, además sus ocho elementos tienen un promedio de índices de discriminación (rit=0.368) estadísticamente significativo y en conjunto, el coeficiente alfa indica una consistencia interna adecuada.

\subsubsection{Estrategias de administración de recursos}

Tabla 6

Estrategias de administración de recursos: Administración del Tiempo

Estrategias de administración de recursos: Administración del Tiempo

\begin{tabular}{|cccccccc|}
\hline $\begin{array}{l}\text { Índice } \\
\text { KMO }\end{array}$ & $\begin{array}{c}\text { Test de Bartlett } \\
\text { (sig) }\end{array}$ & Factores & $\begin{array}{c}\text { \% varianza } \\
\text { explicada }\end{array}$ & Items & Saturación & rit & alfa \\
\hline 0.57 & 0.001 & 1 & 45.64 & A54 & 0.724 & 0.268 & \\
& & & & A73 & 0.703 & 0.251 & 0.42 \\
& & & & A77 & 0.592 & 0.204 & \\
\hline
\end{tabular}

Estrategias de administración de recursos: Constancia

\begin{tabular}{|lccccccc|}
\hline $\begin{array}{l}\text { Índice } \\
\text { KMO }\end{array}$ & $\begin{array}{c}\text { Test de Bartlett } \\
\text { (sig) }\end{array}$ & Factores & $\begin{array}{c}\text { \% varianza } \\
\text { explicada }\end{array}$ & Items & Saturación & rit & alfa \\
\hline 0.818 & 0.001 & 1 & 36.52 & A37 & 0.592 & 0.408 & \\
& & & & A44 & 0.505 & 0.333 & \\
& & & & A65 & 0.647 & 0.452 & \\
& & & A79 & 0.476 & 0.301 & 0.697 \\
& & & A45 & 0.674 & 0.481 & \\
& & & A51 & 0.606 & 0.416 & \\
\hline
\end{tabular}

Estrategias de administración de recursos: Búsqueda de ayuda

\begin{tabular}{|lccccccc|}
\hline $\begin{array}{l}\text { Índice } \\
\text { KMO }\end{array}$ & $\begin{array}{c}\text { Test de Bartlett } \\
\text { (sig) }\end{array}$ & Factores & $\begin{array}{c}\text { \% varianza } \\
\text { explicada }\end{array}$ & Items & Saturación & rit & alfa \\
\hline 0.615 & 0.001 & 1 & 40.622 & A50 & 0.74 & 0.375 & \\
& & & & A56 & 0.73 & 0.361 & \\
& & & & A62 & 0.49 & 0.212 & 0.506 \\
& & & & A67 & 0.552 & 0.288 & \\
\hline
\end{tabular}

La tabla 6 muestra que a las tres escalas les subyace un solo factor o variable latente. Factores estadísticamente significativos como lo señala la media de las saturaciones de 
reactivos: 0.673 para Administración del tiempo; 0.592 para Constancia; 0.628 para Búsqueda de Ayuda, pero, por otro lado, el análisis psicométrico indica que la más débil de estas escalas es Administración del tiempo al tener en promedio un índice de discriminación (rit promedio) de 0.241, el segundo más bajo entre todas las escalas después de Orientación a metas externas. Los coeficientes alfa no alcanzan las cuantías de las escalas de estrategias cognitivas y metacognitivas, siendo el de valor más bajo el de Administración del Tiempo.

\subsection{SÉCCION ESTRATEGIAS DE APRENDIZAJES :Intercorrelación de las escalas}

Tabla 7

\begin{tabular}{|c|c|c|c|c|c|}
\hline & Elabora Organiza & Metacogni & Adm Tiempo & Constan & Ayuda \\
\hline Elabora & $0,622 * *$ & $0,552^{* *}$ & $-0,143 * *$ & $0,513 * *$ & $0,325 * *$ \\
\hline Organiza & -------- & $0,625^{* *}$ & $-0.084 * *$ & $0,552 * *$ & $0,338 * *$ \\
\hline Metacogni & & -------- & -0.045 & $0,553 * *$ & $0,400 *$ \\
\hline Adm Tiempo & & & -------- & 0.022 & $-0,136^{*}$ \\
\hline Constan & & & & -------- & $0,357 * *$ \\
\hline Ayuda & & & & & -------- \\
\hline
\end{tabular}

A diferencia de las escalas de la sección Motivación, las escalas de Estrategias de aprendizaje tienen intercorrelaciones más elevadas y en su mayoría estadísticamente muy significativas ( $\mathrm{p}>0.01$ ). La excepción es la escala Administración del tiempo que si bien tiene coeficientes estadísticamente muy significativos, en cuanto a su tamaño estos coeficientes indican correlaciones que van de nulas a débiles, lo que en términos prácticos significa que no comparte varianza o tiene poco de común con las demás escalas (Elaboración, Organización, Metacognición, Constancia y Búsqueda de ayuda) las que por otra parte alcanzan intercorrelaciones de tamaño "moderado", lo que significa que comparten una parte de varianza significativa, que tienen bastante de común, pero a la vez constituyen aspectos diferentes y unidades psicológicamente significativas.

\section{CONCLUSIONES:}

1. Se ha logrado la elaboración de un cuestionario de motivación y estrategias de aprendizaje basado fundamentalmente en el modelo de aprendizaje autorregulado de Pintrich y DeGreoot y en las modificaciones de Roce y de colaboradores.

2. El cuestionario tiene dos secciones: motivación y estrategias de aprendizaje, Ambas secciones tienen seis escalas que engloban a 85 reactivos.

3. Con un enfoque intrapruebas las escalas del cuestionario demuestran poseer validez de contenido y de construcción, y consistencias internas bastante aceptables.

4. Es conveniente realizar estudios más amplios con criterios externos para establecer con mayor amplitud las características psicométricas del cuestionario. 


\section{BIBLIOGRAFÍA}

Alarcón, R. (1991): Métodos y diseños de investigación del comportamiento. Lima, Perú: Universidad Peruana Cayetano Heredia. Fondo Editorial.

Aliaga, J., Ponce, C., Bernaola, E., \& Pecho, J. (2001). Características psicométricas del inventario de autoevaluación de la ansiedad ante exámenes (Idase). Paradigma (2, 1 ).35-52.

AUSUBEL, D, NOVACK, J. \& HANESIAN, H.( 1983). Psicología educative: un punto de vista cognoscitivo. México, DF., México: Trillas.

Beltrán, J. (1983): Intervención psicopedagógica. Madrid, España: Pirámide.

Cano, E. (1997): Estrategia metacognitiva y cognitivas en el aprendizaje: estudio en alumnos de quinto de secundaria de nivel socioeconómico alto y medio alto de Lima Metropolitana. Tesis de Maestría no publicada. Pontificia Universidad Católica, Lima, Perú.

Capella, J. \& Sánchez Moreno, G. (1999). Aprendizaje y constructivismo. Lima, Perú: Massey and Vannier.

Comrey, A. (1985). Manual de Análisis Factorial. Madrid, España: Cátedra.

Dávila, S.(2000). El aprendizaje significativo. Esa extraña expresión (utilizada por todos y comprendida por pocos). [Online] Contexto Educativo, 48 párrafos. Disponible en: http://contexto-educativo.com.ar/2001/7/nota-08htm.

Díaz Barriga, F. \& Hernández, G. (1998). Estrategias docentes para un aprendizaje significativo. Una interpretación constructivista. México D. F., México: Mc GrawHill.

Elorza, H. (1987). Estadística para ciencias del comportamiento. México, DF, México: Harla.

Hernández, R.; Fernández, C. \& Baptista, P. (1997). Metodología de la Investigación. México DE, México: Mc Graw Hill.

Gonzáles-Cabanach, R Valle, A, Súarez, J y Femández, A. (1999): Un modelo integrador explicativo de las relaciones entre metas académicas, estrategias de aprendizaje y rendimiento académico. Revista de Investigación Educativa" Vol. 17. W 1: 47-70.

Martínez, R. \& Galán, E (2000). Estrategias de aprendizaje, motivación y rendimiento académico en alumnos universitarios. Revista de Educación y Orientación Profesional (REOP). Vol. 11, № 19, ler. Semestre; 35-50

Mendo, J. (1998). Paradigmas en Educación. Educación Superior. Año I, 1,7-20.

Monereo, C. (1993). Las estrategias de aprendizaje: procesos, contenidos e 
interacción. Barcelona, España: Doménech.

Morales, P. (1988). Medición de actitudes en psicología y educación. Construcción de escalas y problemas metodológicos. San Sebastián, España: Tarttalo.

Nisbeth, J.y Shucksmith, J. (1987). Estrategias de aprendizaje. Madrid, España: Santillana. Núñez, J, Gonzáles-Pienda, J.; García, S. \& Gonzáles-Pumariega, S. (1996). Motivación en el ámbito univeisitario: concepto de inteligencia, metas de estudio, elección de tareas y aproximaciones al aprendizaje. Revista de Educación $\mathrm{N}^{\circ}$ 310: 337.360.

Pintrich, P. y DeGroot, E (1990): "Motivacional and Self-Regulated Leaming Components of Classroom Academic Performance". Journal of Educational Psychology, 82( 1), 33-40.

Pintrich, P. R., Smith, D.A., García, T. y Mckeachie, W.J. (1991). Reliability and Predictive Validity of the Motivational Strategies for Learning Questionnaire (MSLQ). Educational and Psycological Measurement, 53, 801-813.

Roces, C.; Gónzales - Pienda, J. A.; Núñez, S.; Gonzáles - Pumariega, S.; García, M \& Alvarez, L. (1999). Relaciones entre motivación, estrategias de aprendizaje y rendimiento académico en estudiantes universitarios. Mente y conducta en situación educativa. Revista Electrónica del Departamento de Psicología. Universidad de Valladolid [Online]1 (1), 61 párrafos. Disponible en: http://www.uva.es/psicologia/01014150.htm [6 enero 2002]

Roces, C., Gonzáles, M. C. \& Touron, J, (1997). Expectativas de aprendizaje y rendimiento de los alumnos universitarios. Revista de Psicología de la Educación, 22, 99 123.

Román, J. M. y Gallego, S (1994): ACRA" Escalas de Estrategias de Aprendizaje. Madrid, España: TEA.

Sierra Bravo, R.(1994). Técnicas de investigación social. Teorías y ejercicios. Madrid, España: Paraninfo. 